\title{
A SCREENING TECHNIQUE FOR ESTABLISHING THE STABILITY OF METAL FILM RESISTORS
}

\author{
A. P. GOSWAMI, L. SATYANARAYANA and N. V. M. SIVAJI \\ $R \& C$ Division, Electronics Corporation of India Ltd., Hyderabad-500 762, India
}

(Received January 8, 1977; in final form June 15, 1977)

\begin{abstract}
A technique has been designed whereby stability data for metal film resistors may be predicted within 24 hours. Regressional analysis and statistics are used in the technique.
\end{abstract}

\section{INTRODUCTION}

Stability and reliability of passive components in sophisticated instruments are considered to be of prime importance by defence, space and communication users. Such customers prefer the manufacturer to quote both of these parameters whose measurement is a cumbersome and time consuming process. A good stability inherently leads to better reliability figures. It must be stressed here that in the large scale manufacture of components, especially resistors, conditions cannot always be kept homogenous and thereby if not properly checked can lead to inferior qualities in performance. It is now well known that stability of a resistor depends essentially on the nature of the film coating and the subsequent annealing treatments. Thus a ridiculous situation would arise when in order to establish the stability of samples of resistors made from each batch after vacuum coating they would have to be subjected to 1000 hours of electrical ageing before being processed for actual production. For precision metal film resistors, the resistance deviation after electrical ageing by dissipating the actual wattage at $125^{\circ} \mathrm{C}$ or twice the rated at $70^{\circ} \mathrm{C}$ for 1000 hours in case of lower wattage types should be $\leqslant 0.5 \%$ as per MIL, DEF, JSS (Indian Defence Specification) or Indian Standards. However, in case of general purpose metal film resistors, which are in vogue today, the permissible change is $1 \%$. In order to establish the stability results quickly, we have designed a technique whereby this data could be predicted within 24 hours. This method has been made possible by the use of regressional analysis and statistics.

\section{DESIGN OF EXPERIMENT}

A large quantity of semi-finished resistors of wide range of values and low TCR $\left(\approx 50 \mathrm{ppm} /{ }^{\circ} \mathrm{C}\right)$ were selected at random from the shop. It was, therefore, presumed that the samples were a representation of the manufacturing process and the selection was unbiased. These were then electrically aged as per JSS methods to evaluate their stability. The change in resistance was measured initially, after 24 hours and subsequently after 100 to 200 hour intervals of time. During measurements the ambient temperature was maintained constant $\left(27 \pm 2^{\circ} \mathrm{C}\right)$ to eliminate the chance of the TCR masking the actual change in resistance. ESI decade boxes of $\pm 0.01 \%$ accuracy and a sensitive Bruel and Kjaer deviation bridge (Model 1521) were used for this purpose. Because of the close measuring tolerances after initial loading the same decade boxes, bridge and jigs were used. The resistance values selected were $4 \mathrm{kohm}, 10 \mathrm{kohm}$, $40 \mathrm{kohm}, 60 \mathrm{kohm}, 150 \mathrm{kohm}$ and $220 \mathrm{kohm}$ each of about 30 pieces and also embracing a wide range of sheet resistivities.

\section{COMPUTATION OF REGRESSION EQUATION AND ANALYSIS}

The theme in this experiment is to find a correlation, if any, between the change in resistance after 24 hours of electrical ageing $\left(\Delta R_{24}\right)$ and that which would be obtained if the same resistors were subjected to 1000 hours of loading $\left(\Delta R_{1000}\right)$. Of the 200 resistors studied, Table I shows the values for $\Delta R_{24}$ 
TABLE I

\begin{tabular}{|c|c|c|c|c|c|}
\hline Observation no. & $\Delta R_{24}$ & $\Delta R_{1000}$ & Observation no. & $\Delta R_{24}$ & $\Delta R_{1000}$ \\
\hline 1 & .02 & .18 & 33 & .04 & .62 \\
\hline 2 & .07 & .55 & 34 & .02 & .38 \\
\hline 3 & .03 & .19 & 35 & .03 & .48 \\
\hline 4 & .05 & .37 & 36 & .01 & .06 \\
\hline 5 & .06 & .60 & 37 & 0 & .15 \\
\hline 6 & .03 & .27 & 38 & 0 & .22 \\
\hline 7 & .04 & .41 & 39 & .01 & .13 \\
\hline 8 & .06 & .62 & 40 & 0 & .20 \\
\hline 9 & .04 & .29 & 41 & .01 & .12 \\
\hline 10 & .07 & .80 & 42 & 0 & .17 \\
\hline 11 & .05 & .55 & 43 & .01 & .40 \\
\hline 12 & .02 & .32 & 44 & 0 & .23 \\
\hline 13 & .1 & 1.00 & 45 & 0 & .16 \\
\hline 14 & .01 & .22 & 46 & .02 & .53 \\
\hline 15 & .02 & .40 & 47 & .01 & .12 \\
\hline 16 & .02 & .35 & 48 & .01 & .22 \\
\hline 17 & .02 & .35 & 49 & .01 & .42 \\
\hline 18 & .03 & .35 & 50 & .01 & .25 \\
\hline 19 & .02 & .30 & 51 & .01 & .41 \\
\hline 20 & & .25 & 52 & .01 & .34 \\
\hline 21 & .02 & .35 & 53 & .01 & .23 \\
\hline 22 & .03 & .40 & 54 & .01 & .38 \\
\hline 23 & .04 & .35 & 55 & .01 & .21 \\
\hline 24 & .02 & .15 & 56 & .08 & .86 \\
\hline 25 & .01 & .18 & 57 & .02 & .54 \\
\hline 26 & .02 & .28 & 58 & .02 & .30 \\
\hline 27 & .01 & .15 & 59 & .09 & .94 \\
\hline 28 & 0 & .22 & 60 & .01 & .31 \\
\hline 29 & .01 & .20 & 61 & 0 & .18 \\
\hline 30 & .02 & .35 & 62 & .04 & .57 \\
\hline 31 & .03 & .41 & 63 & .03 & .41 \\
\hline 32 & .03 & .51 & & & \\
\hline
\end{tabular}

$\Delta R_{24}=\%$ Change in resistance after 24 hours of electrical ageing.

$\Delta R_{1000}=\%$ Change in resistance after 1000 hours of electrical ageing.

and corresponding $\Delta R_{1000}$ for 63 numbers selected at random. To obtain a clear picture both these parameters were plotted on a linear scale as shown in Figure 1. The relation is apparently linear. It may be pointed out here that in a few cases if untoward deviations for $\Delta R_{1000}$ had been observed in the sample, then they could be ignored as outliers. To estimate the best fit equation for the plots, the regression equation is computed by the outline given below. ${ }^{1}$

Let us denote $\Delta R_{24}$ and $\Delta R_{1000}$ as ' $X$ ' and ' $Y$ '. For the regression equation the variance due to $X, Y$ and the co-variance (due to $X$ and $Y$ ) are calculated as:

$$
S_{x x}=\Sigma x_{i}^{2}-\frac{\left(\Sigma x_{i}\right)^{2}}{n}
$$

$$
\begin{aligned}
& S_{y y}=\Sigma y_{i}^{2}-\frac{\left(\Sigma y_{i}\right)^{2}}{n} \\
& S_{x y}=\Sigma x_{i} y_{i}-\frac{\Sigma x_{i} \Sigma y_{i}}{n}
\end{aligned}
$$

where $i=1,2,3, \ldots, n$ and $n$ is the total number of observations. ' $x$ ' and ' $y$ ' are individual observations for $\Delta R_{24}$ and $\Delta R_{1000}$ respectively. The regression equation is then given as

$$
\begin{aligned}
& (y-\bar{y})=\frac{S_{x y}}{S_{x x}}(x-\bar{x}) \\
& \text { where } \bar{y}=\frac{\Sigma y_{i}}{n} \text { and } \bar{x}=\frac{\Sigma x_{i}}{n}
\end{aligned}
$$




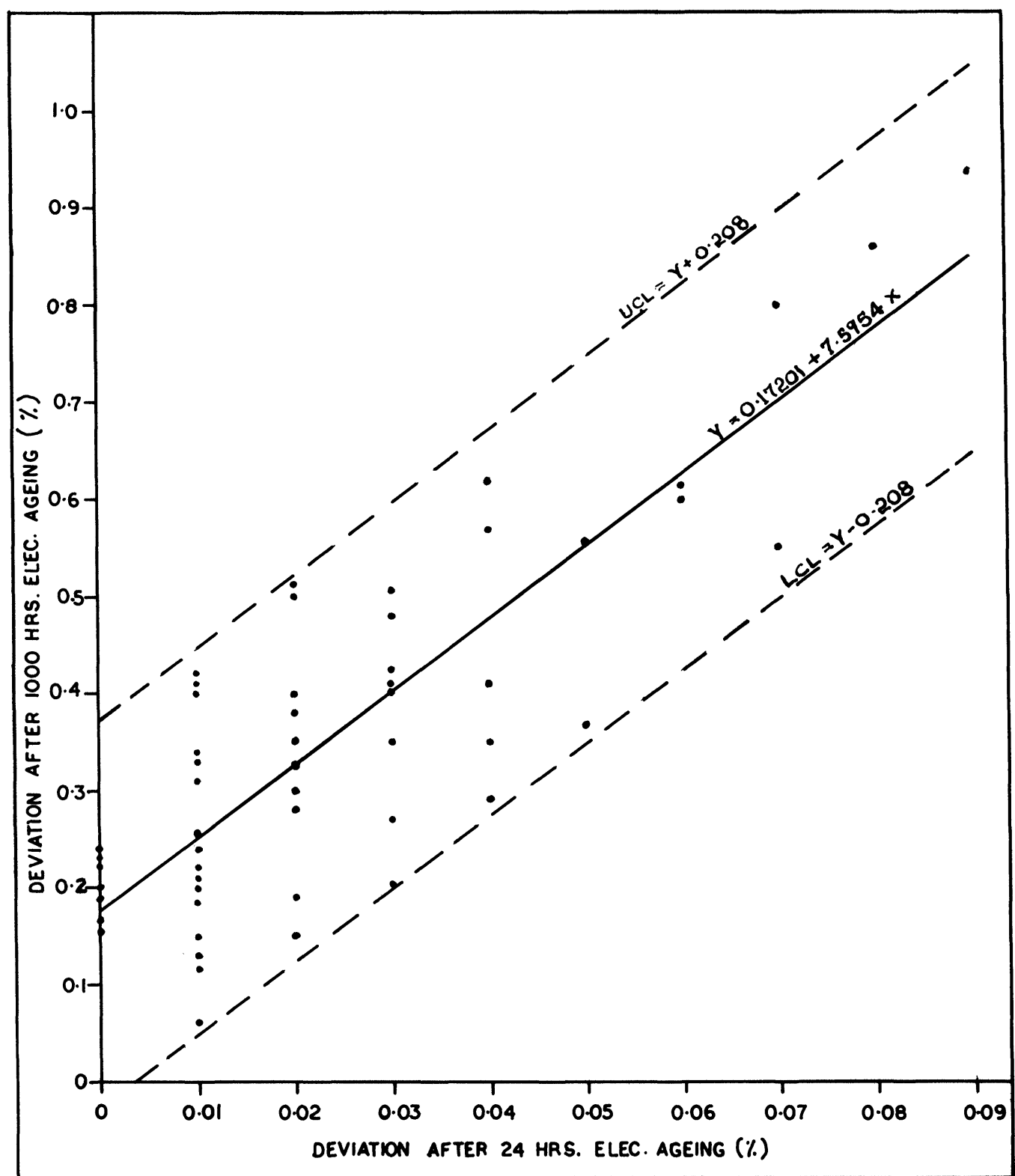

FIGURE 1 Resistance deviation after 1000 hours of electrical ageing ( $\Delta R_{1000}$ ) against resistance deviation after 24 hours of electrical ageing $\left(\Delta R_{24}\right)$.

The test for the strength of linearity in the above equation is obtained by computing the coefficient of correlation $(r)$ where

$$
r=\frac{S_{x y}}{S_{x x} S_{y y}}
$$

and testing for its significance by the ' $t$ ' statistic for $(n-2)$ degrees of freedom where $t$ is given as

$$
t=\frac{r \sqrt{n-2}}{1-r^{2}}
$$

If this computed value of ' $t$ ' is larger than the 
tabulated one for $(n-2)$ degrees of freedom then corresponding to that level of significance the correlation is significant and the computed equation in Eq. (4) is linear. If on the other hand the table value is larger than the computed ' $t$ ' then the vice versa holds true. In general $-1 \leqslant r \leqslant 1$ and thus positive and negative correlation could be obtained.

Using this relationship the values of the dependent variable $(Y)$ can be predicted from the independent variable $(X)$. However, the estimated values of $Y$ are subject to errors which include uncontrollable chance causes as well as measurement errors. The standard error of the estimate gives a measure of the divergence of the actual values of the dependent variable from their estimated value. This is given by

$$
\sigma_{e}= \pm \frac{\sqrt{S_{y y}\left(1-r^{2}\right)}}{n-2}
$$

For a $96.4 \%$ confidence level in the computed linear equation the error is incorporated in Eq. (4) as

$$
(y-\bar{y})=\frac{S_{x y}}{S_{x x}}(x-\bar{x}) \pm 2 \sigma_{e}
$$

This refined equation would then give a spread or envelope whose upper and lower control limits would be

$$
\text { U.C.L. }(y-\bar{y})=\frac{S_{x y}}{S_{x x}}(x-\bar{x})+2 \sigma_{e}
$$

and

$$
\text { L.C.L. }(y-\bar{y})=\frac{S_{x y}}{S_{x x}}(x-\bar{x})-2 \sigma_{e}
$$

\section{RESULTS}

From the sample's data in Table I, we obtain the parameters required for the computation as follows:

$$
\begin{aligned}
& \Sigma x_{i}=1.48 ; \bar{x}=0.02349 ; \Sigma x_{i}{ }^{2}=0.0670 . \\
& \Sigma y_{i}=22.08 ; \bar{y}=0.35042 ; \Sigma y_{i}{ }^{2}=10.2618 \\
& \Sigma x_{i} \Sigma y_{i}=0.7635 ; \text { where } i=1,2,3, \ldots, 63 . \\
& \text { Similarly } S_{x x}=0.03223, S_{y y}=2.5233 \\
& S_{x y}=0.2448 .
\end{aligned}
$$

The correlation coefficient ' $r$ ' obtained was 0.8584 (from Eq. (5)) and the ' $t$ ' statistic was computed as 25.48. Since this is larger than the table value of 2.00 obtained for $61[(n-2)]$ degrees of freedom for 95\% level of confidence, the correlation coefficient ' $r$ ' is strongly significant and the linear equation becomes

$$
Y=0.17201+7.5954 X
$$

Substituting the original notations we have

$$
\Delta R_{1000}=0.17201+7.5954 \Delta R_{24}
$$

The standard error estimated is 0.104 and the equation can be refined as

$$
\begin{aligned}
\Delta R_{1000}= & 7.5954 \Delta R_{24}+0.17201 \\
& \pm 0.208
\end{aligned}
$$

For $96.4 \%$ confidence level, the control limits then become

$$
\begin{aligned}
\text { U.C.L. } \Delta R_{1000}= & 7.59540 \Delta R_{24} \\
& +0.38001
\end{aligned}
$$

and

$$
\begin{aligned}
\text { L.C.L. } \Delta R_{1000}= & 7.5954 \Delta R_{24} \\
& -0.03599
\end{aligned}
$$

Figure 1 shows the linear relationship between $\Delta R_{1000}$ and $\Delta R_{24}$ with the upper and lower control limits.

\section{DISCUSSIONS}

We have shown by statistical technique of linear regression that a positive correlation between $\Delta R_{24}$ and $\Delta R_{1000}$ does exist and the relation between them is given by the linear equation of the form expressed in Eq. (10). The upper and lower control limits (see Eq. (11)) predict with $96.4 \%$ confidence the estimate for $\Delta R_{1000}$ after 24 hours of electrical ageing $\left(\Delta R_{24}\right)$. From Figure 1, it can be seen that for the resistors to have a stability of $0.5 \%$ after 1000 hours life test, the initial change in 24 hours should be preferably $\leqslant 0.02 \%$. Similarly for general purpose metal film resistors wherein a stability of $1 \%$ is desired, $\Delta R_{24}$ could be as high as $0.08 \%$. Further, this equation is independent of the resistance values and sheet resistivity. 


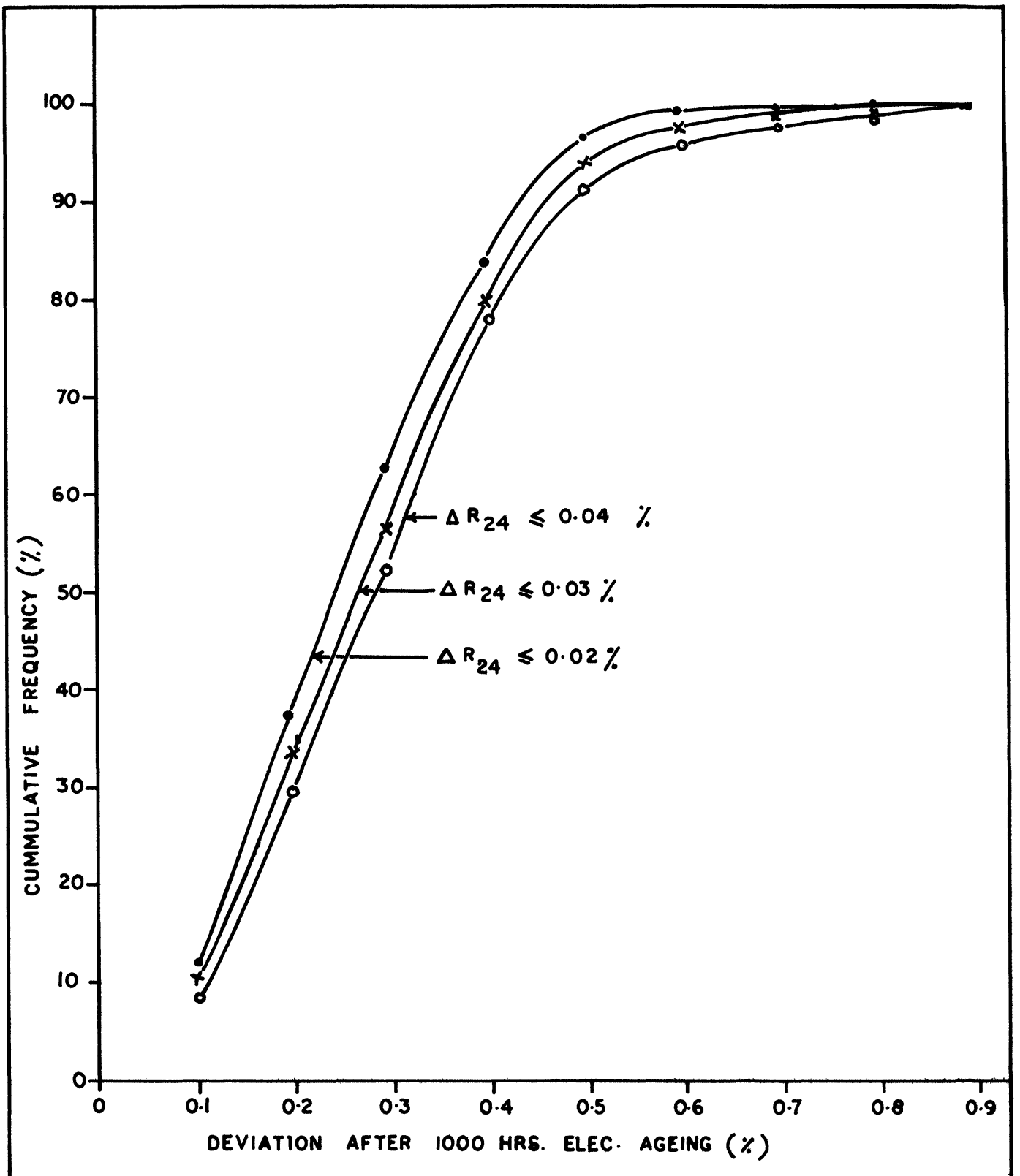

FIGURE 2 Cumulative frequency response against resistance deviation after 1000 hours of electrical ageing.

To test the validity of this theory, we have selected resistors whose initial changes after 24 hours of electrical ageing were $\leqslant 0.02 \%, \leqslant 0.03 \%$ and $\leqslant 0.04 \%$. Figure 2 shows the cumulative frequency distribution expressed as percentages for $\Delta R_{1000}$ for resistors so selected. It can be clearly seen that only
$3 \%$ rejection is observed when the resistors are screened for $\Delta R_{24} \leqslant 0.02 \%$ which is within the permissible limits expounded from theoretical calculations. For $\Delta R_{24} \leqslant 0.03 \%$ the rejection is about $6 \%$ and higher in case of $\Delta R_{24} \leqslant 0.04 \%$. The quantity of resistors so screened was about 160 


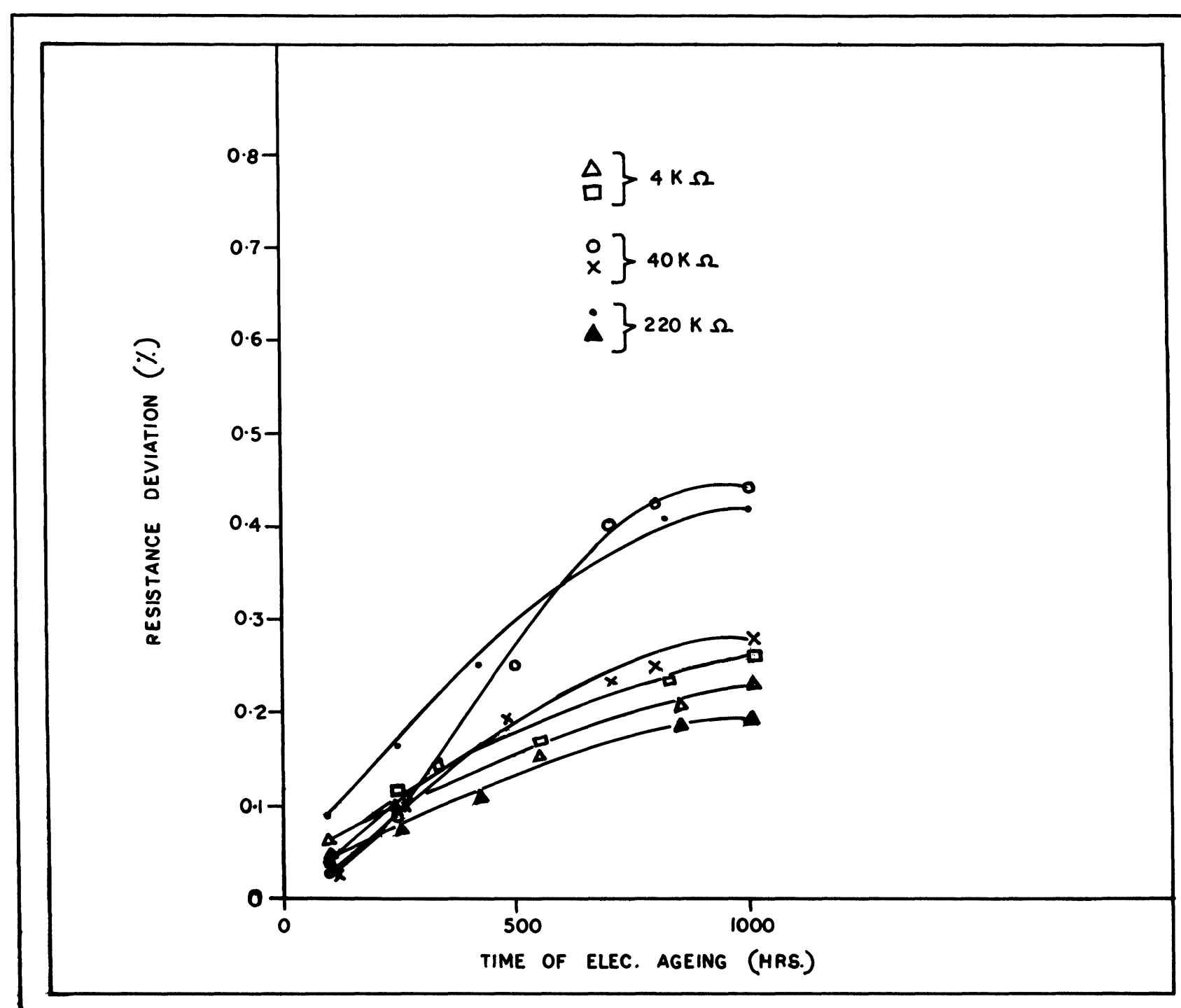

FIGURE 3 Resistance deviation against time of electrical ageing.

embracing a wide range of values. However, if the lot size was still larger we are sure that a much better representation would have been obtained.

Collection of this data is now in progress.

There may be some apprehension in using this technique if a change in sign for resistance deviation was observed either during the initial 24 hours or after the final 1000 hours of electrical ageing. Although such deviations cannot be ruled out, we would like to mention that such changes are probably due to process conditions. In all our studies as well as regular production we have found the resistance deviation during electrical ageing always to be positive. If a change of sign does occur then a relation has to be found either by regression or otherwise taking into account such deviations. We would like to stress here that the linear equation obtained by us may not be universal, but that it is possible to correlate the two ageing parameters. The control limits derived here are true only for our process conditions and extrapolation or interpolation of them beyond the range are not valid. The requirement for $\Delta R_{24} \leqslant 0.02 \%$ for $0.5 \%$ stability precision resistors is not unrealistic in the sense that the linear 
equation generated takes into account all measurement errors and chance causes during the processing. There is little probability of the change in resistance due to temperature masking the actual resistance deviation as the TCR is very low $\left(25\right.$ and $50 \mathrm{ppm} /{ }^{\circ} \mathrm{C}$ ) for precision resistors. In case of general purpose resistors whose TCR is around $100 \mathrm{ppm}$ and stability is $1 \%, \Delta R_{24}$ could be as high as $0.08 \%$.

The control chart prepared in Figure 1 can now serve a dual purpose in the manufacture of metal film resistors:

1) As a method to screen components whose stability would be as per specifications and give high reliability figures.

2) This can also be used to assure that all products would have the required stability performance by enforcing the screening technique after vacuum metallisation stage. Acceptance tests for each metallised batch could be conducted with appropriate $\mathrm{AQL}$, so that $\Delta R_{24} \leqslant 0.02 \%$ and $\Delta R_{24} \leqslant$ $0.08 \%$ could be taken as resistance deviation acceptances for $0.5 \%$ and $1 \%$ stability resistors respectively before being sent for further processing.

However, as a precaution the linear equation must be tested from time to time to see that no untoward deviation from the control limits occurred. Once the confidence in this has been built up, no further monitoring of the limits is required.

\section{APPLICATION FOR RELIABILITY SCREENING}

At the time of this communication reliability figures for 8,000 hours were not available. However, as can be seen from Figure 3, deviation in resistance value diminishes after about 700 hours of ageing. An extrapolation of the graphs to 8000 hours would show the deviation to be well within the permissible limit of $2 \%$, as catastrophic failures at this stage are not likely to occur. On obtaining the data for such resistance deviation after 8,000 hours, a regression analysis could be made to find a correlation if any, between this and $\Delta R_{24}$ and control limits can be set up as outlined in the technique above. Work is now under progress for this study.

\section{ACKNOWLEDGEMENT}

We are extremely grateful to Prof. D. S. Campbell of Loughborough University of Technology and the referees for their valuable suggestions in this work. We also wish to acknowledge the assistance received from Sri P. K. Basu of SQC Unit of the Indian Statistical Institute, Hyderabad.

\section{REFERENCE}

1. Course on "Regression analysis and design of experiments", Indian Statistical Institute, Statistical Quality Control Unit, Hyderabad (1976). 

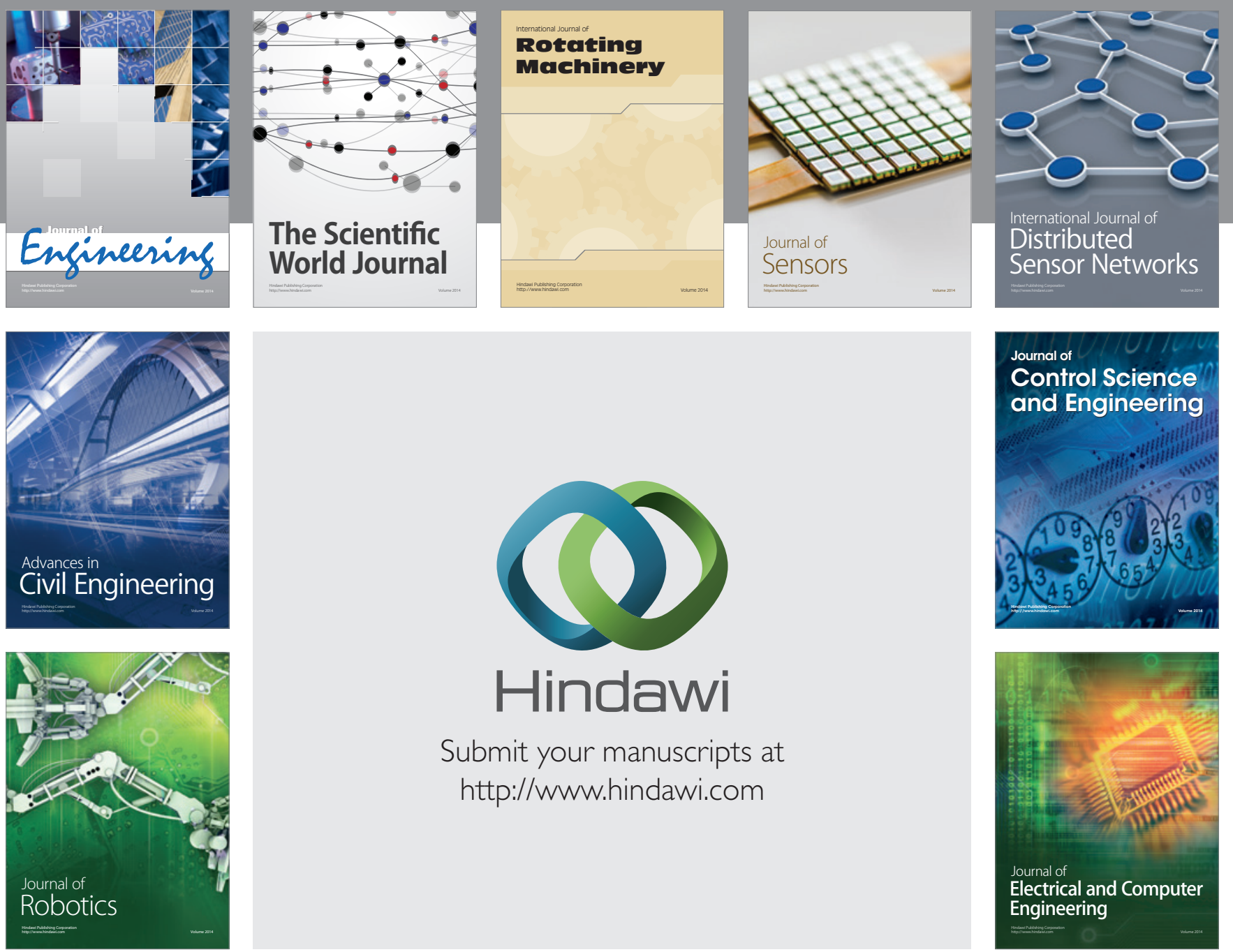

Submit your manuscripts at

http://www.hindawi.com
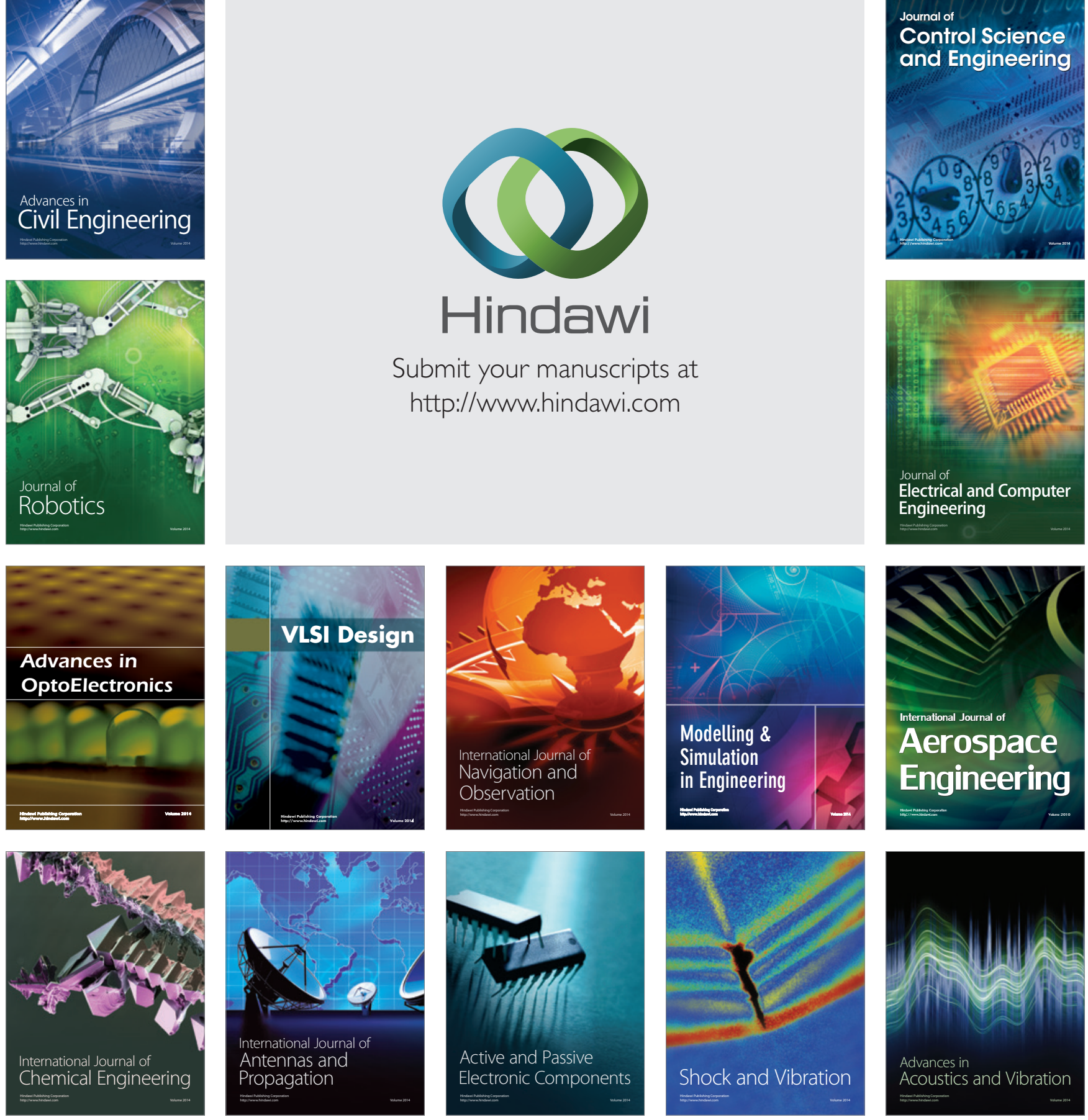